\title{
Usefulness and Limitations of Skin Explants to Assess Laser Treatment
}

\author{
Hong-il Cho ${ }^{1}$ \\ Chong Hyun Won ${ }^{1}$ \\ Sung Eun Chang ${ }^{1}$ \\ Mi Woo Lee ${ }^{1}$ \\ Gyeong-Hun Park ${ }^{2}$
}

\footnotetext{
${ }^{1}$ Department of Dermatology, Asan Medical Center, University of Ulsan College of Medicine, Seoul, Korea

${ }^{2}$ Department of Dermatology, Dongtan Sacred Heart Hospital, Hallym University College of Medicine, Hwaseong, Korea
}

To overcome the ethical issues associated with performing clinical trials or obtaining patient's skin tissues, there have been many attempts to develop appropriate models to study skin physiology, such as the use of skin explants to assess the effects of laser treatment. Skin explants are produced by processing skin tissue obtained from an excision. Contaminants and subcutaneous fat tissue are removed from the tissue, which is then cultured. Such processed tissue lacks blood circulation and nerve innervation, and is difficult to be preserved over a prolonged period of time. However, due to the similarities between skin explants and live skin tissues in terms of structure and physiological factors, such explants are thought to be useful as skin models to study the effects of laser treatment. Our previous studies showed that, following exposure to actual fractional laser, skin explants have very similar histological and molecular biological changes to those seen in the initial in vivo stages. However, with time, the differences between skin explants and live skin tissues become increasingly apparent. Therefore, skin explant models may be improved, such as by optimizing the culture conditions. In our present review, we examined the usefulness and limitations of skin explants to study the effects of laser therapy based on our own previous experience and the existing literature.

\section{Key words}

Skin explant; Laser study; Fractional laser 


\section{INTRODUCTION}

Various types of laser are now being used in a number of medical applications. In the dermatology field, laser therapy has been widely used to treat pigment disorders, vascular lesions, and scars. The efficacy of such laser treatments has been verified repetitively in various clinical trials and in clinical practice. However, few studies have examined the mechanism underlying laser efficacy and the molecularbiological level changes resulting from the interactions between lasers and tissues. This low number of studies is mainly attributable to difficulties in the methodology, especially in the difficulty of securing samples. Multiple extracts of patients' skin tissue are required to study changes in tissues. However, the process of extracting skin tissues through repeated biopsies at baseline and at subsequent time points can be difficult due to ethical and also esthetic considerations. To overcome these issues, there have been numerous attempts to develop skin models to replace the need for live human skin tissues. Of these, skin explants have been widely used in studies of wound healing and the inflammatory process. In our current review, we examined the usefulness and limitation of skin explants to investigate the effect of laser treatment.

\section{ADVANTAGES AND DISADVANTAGES OF SKIN EXPLANTS}

\section{Skin models for dermatological studies}

There has been significant progress in developing various skin models for dermatological studies or for the testing of cosmetics and medicines. Such skin models include mono-cellular culture models, co-culture models, organotypic models, and in vivo and in virtuo models. While in vivo models are the most physiologic model system, limitations include legal and ethical issues as well as the considerable cost and time required. 'In vivo animal testing has been widely used to evaluate the efficacy of drugs. However, the recent increase in ethical awareness and legal issues encouraging alternatives to animal testing impose limitations on the usage of animal studies. In addition, animal skin tissue differs from that of people in terms of histology and physiology ${ }^{2}$; however, there is no reliable alternative established to replace the use of animal models.

\section{Skin explants}

Skin explants are obtained from skin samples extracted after a skin excision. Contaminants and subcutaneous fat tissue are removed from the samples, and the remaining tissue is then cultivated. The skin explants can either be prepared by separating the epidermis and the dermis, or the entire tissue can be used without separation. ${ }^{1}$ Key culture factors include temperature, light, and humidity. Currently available culture media include DMEM and DMEM/Ham'sF12, 3 , whereas William's E culture medium is used for human scalp tissue, with fetal bovine serum used as a supplement. ${ }^{5}$

\section{Advantages and limitations of skin explants}

Organotypic models, which include skin explants and reconstructed skins, have the advantage of providing a 3-dimensional structure, whilst mono-cellular culture and co-culture models only show two dimensions. In addition, whereas mono-cellular cultures by definition consist of only one type of cell, organotypic models include inter-cellular interactions, such as those between keratinocytes and fibroblasts. In addition, organotypic models are easier to use compared to in vivo models. However, short comings include the lack of circulation and nerve innvervation, which are present in in vivo models, difficulty in controlling sample conditions, and the absence of desquamation. ${ }^{6-9}$

Of these models, skin explants can achieve physiological outcomes due to their distinct characteristics. 'The advantage of skin explant models is the presence of all, or at least most, types of cells, while reconstructed skin contains only fibroblasts, keratinocytes, and melanocytes. ${ }^{8,10,11}$ In addition, skin explants, but not reconstructed skins, contain information on the age and living patterns of the subject le.g., sunlight exposure, treatment history, and allergy). Furthermore, skin explants are cheaper to produce and can be used immediately, while reconstructed skin requires a longer time to prepare and the reconstruction process itself can be very difficult. However, because the properties of skin explants depend entirely on the biopsied tissue, it is difficult to control the characteristics of the samples which are dependent on the age and living patterns of the donors. Reconstructed skin can be used for an extended period of time during the maturation period, which ranges from a few days to a few weeks, while skin explants should be, in general, used within a period of 10 to 14 days based on the culture conditions (Table 1).'

\section{Effects of skin explants}

In spite of the aforementioned shortcomings, skin explants can be used as an alternative to animal models for skin efficacy testing. With more advanced skin explant systems, it may be possible to develop more reliable 
Table 1. Comparison of reconstructed skin and skin explant models

\begin{tabular}{cll}
\hline Model & \multicolumn{1}{c}{ Advantages } & \multicolumn{1}{c}{ Limitations } \\
\hline Reconstructed skin & Use of various conditions & Control of maturation time \\
& Many applications & Lack of appendages \\
& & Lack of innervation and circulation \\
& Difficult and long to produce & High cost of creating and maintaining \\
Skin explant & Ext cell types represented & Impossible to use wanted conditions \\
& Many applications & No control of maturation \\
& Representative of life habits & Short duration \\
& Easy to produce & Dependent on human biopsy \\
& Low cost to generate & Lack of innervation and circulation \\
\hline
\end{tabular}

models to test the whitening effects or anti-aging effects of certain products. As it is possible to measure various functional indicators of the skin to test moisturization, barrier effects, and wound healing, which are difficult to test in animals or in cell culture, skin explants may contribute to expansion of the range of studies to examine the laser-tissue interaction of clinical laser devices.

\section{ADVANTAGES OF USING SKIN EXPLANTS IN LASER STUDIES}

\section{Previous experience in fractional laser studies}

Ex vivo skin models have been used in many studies on the effects of ablative and nonablative fractional lasers. Such studies mainly involve exposing the excised skin samples to lasers in order to evaluate the histological changes that occur immediately after laser treatment, thereby not requiring organotypic culturing of skin samples. ${ }^{12,13}$ The histological changes following laser exposure, as assessed by ex vivo methods, have been found to be nearly the same as those obtained in in vivo tests. Exposure of a skin sample to carbon dioxide fractional laser ex vivo has revealed many microscopic treatment zones and thermal coagulation zones where the epidermis and the dermis were histologically ablated. ${ }^{12}$ The microscopic treatment zones were also observed in a test using a non-ablative fractional laser, which revealed columns of the denatured epidermis and dermis and subepidermal clefting, or the disruption of the dermo-epidermal junctions. ${ }^{14}$ Such histologic changes ex vivo were also nearly the same as those observed in in vivo tests. ${ }^{15,16}$ In addition, the depth and the width of the microscopic treatment zones after non-ablative fractional laser treatment were similar between the ex vivo and the in vivo models. ${ }^{15}$

Another study observed the morphological changes during organotypic culture after ex vivo exposure to a variety of fractional lasers. ${ }^{14}$ Skin explants cannot be maintained over a prolonged period of time particularly in immersed conditions. For this reason, in the study, the samples were cultivated for a prolonged period under stable conditions on the air-liquid interface by wrapping the tissue with agarose, which prevented it from being fully submerged in the culture medium contained in the micropore transwell chamber. These conditions were maintained for 7 days. The data indicated that the immediate changes following laser exposure were similar to those found in in vivo tests for the carbondioxide fractional laser, erbium-doped yttrium aluminum garnet fractional laser, and non-ablative fractional laser. At 1 to 3 days after exposure to the lasers, the epidermis was reepithelialized with the formation of necrotic debris in the upper epidermis, which was reminiscent of the reepithelialization and the microscopic epidermal necrotic debris (MEND) in vivo. However, the formation of MEND in skin explants was not as prominent as in the in vivo findings, and they had not completely extruded until day $7 .^{14,16,17}$ In addition, the time taken for reepithelialization of the skin explants ranged from 1 to 3 days in contrast to the 12 to 24 hours seen in vivo. ${ }^{14,17-19}$ In addition, whilst the epidermis is invaginated into the dermis in vivo as a result of the thickening of the epidermis during wound healing after ablative fractional laser exposures, ${ }^{16,20}$ the thickness of the reepithelialized epidermis in skin explants was thinner than that in the other surrounding epidermal tissues. ${ }^{14}$ As skin explants lack blood circulation, there was no inflammatory cell infiltration, which is observed in vivo. $14,16,17,19$

In addition to morphologic changes, other studies have focused on molecular or functional changes in skin explants after fractional laser exposure. ${ }^{21}$ Microarray analysis was conducted to identify the gene expression profile of skin explants 2 to 24 hours after exposure to a carbon dioxide fractional laser. The results revealed 
significantly upregulated expression of cysteine-rich angiogenic inducer 61 at 2 hours and Wnt5a at 24 hours, and these findings were confirmed by real-time RT-PCR. In addition, using laser scanning confocal microscopy, the expression levels of the proteins were also found to be increased in vivo. However, there was a discrepancy in the expression levels of some of the genes, such as heat shock protein 90, between the skin explants and the in vivo data.

\section{Limitations and future perspectives of skin explants}

Based on previous studies, it appears that skin explant experiments can mirror in vivo fractional laser studies. In particular, such benefits are expected to be most prominent in studies concerning the initial changes after exposure to laser, considering the relatively short maintenance and the absence of blood circulation in the skin explants. However, the core skin changes that occur following fractional laser exposure are thought to be associated with the remodeling of collagen in the dermis. Skin explants cannot be maintained for a sufficient time to show such histologic changes. In addition, there is a gradual degeneration of the dermal collagen. These factors could undermine the reliability of the changes observed in the dermis of skin explants. In addition, the human skin tissues used in previous studies have been mainly obtained from the abdomen. However, skin tissue of the face and the abdomen may differ in the distribution of sebaceous glands and hair follicles. In addition, because these skin samples are obtained during surgery, the amount obtained at each excision is limited. Another hurdle is that the extracted skin parts must be processed in a timely manner, meaning that researchers must carefully schedule their studies to account for processing time (Table 2). The skin tissues of Yorkshire pigs or Yucatan mini-pigs are known to have similar characteristics to that of human skin, and, therefore, in vivo pig models may also be a useful alternative of skin explants to study fractional laser effects. ${ }^{22,23}$

Table 2. Advantages and limitations of skin explants in laser studies

\begin{tabular}{ll}
\hline \multicolumn{1}{c}{ Advantages } & \multicolumn{1}{c}{ Limitations } \\
\hline Reflects the 3-dimensional structure of human skin & Difficult to maintain for a long time \\
Presence of hair follicles, sweat glands, and other skin components & No blood circulation \\
Virtually all cellular components of the skin & Difficult to assess changes in the dermis \\
Reflects the characteristics of the donors and originating parts & Difficult to obtain a sufficient amount of skin sample from the face \\
Cheaper & Difficult to organize the study schedule \\
Useful in assessing the initial changes after exposure to laser & No inflammatory cell infiltration \\
& Difficult to observe later-stages of laser-induced changes \\
\hline
\end{tabular}

A

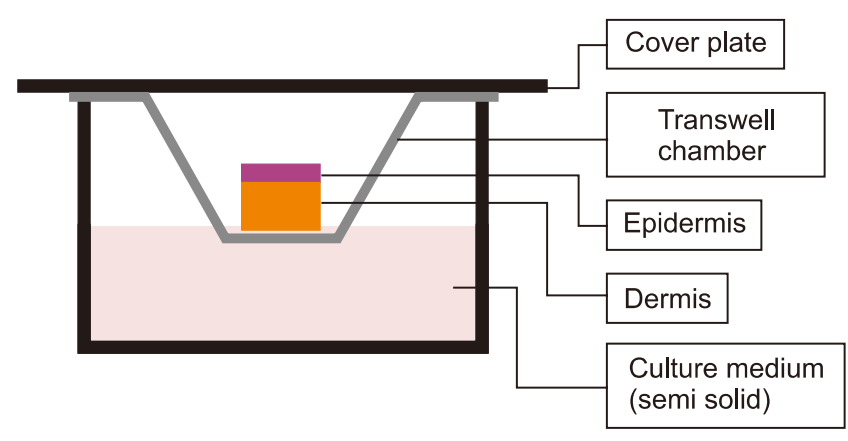

B

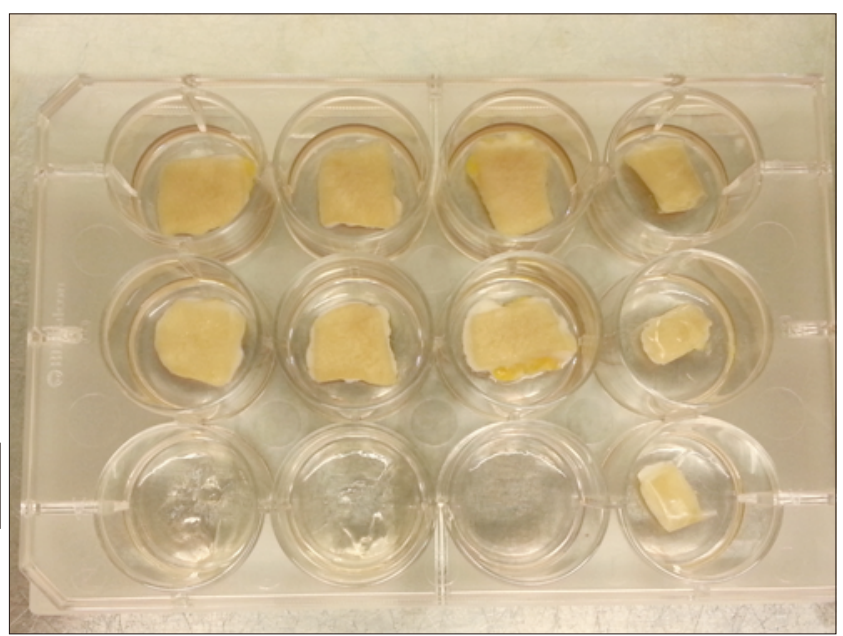

Fig. 1. Skin explant models. (A) Skin explants adapted in a transwell chamber. (B) Skin explants in a 12-well culture dish. 


\section{CULTURE CONDITIONS FOR SKIN EXPLANTS}

There are many important factors to consider when performing skin explant culture (Fig. 1). These factors depend on the duration of the culture, and different approaches should be used in accordance with the purpose of the culture. For short-term cultures, the tissue can be submerged in the growth medium. One method of doing this is to prepare a skin fragment, remove the fat tissue from underneath, and wash for 10 minutes in serum-free keratinocyte culture medium (Keratinocyte SFM; Life Technologies, Paisley, UK). The entire tissue is then placed on a multiwellplate (Costar, Cambridge, MAl and cultivated for 2 days in $1 \mathrm{ml}$ keratinocyte SFM at $37^{\circ} \mathrm{C}, 5 \% \mathrm{CO}_{2}{ }^{24}$ For a long-term culture, the tissue should be cultured on the air-liquid interface. To do this, a 2-mm hole is made on a transwell filter (pore size 0.75 $\mu \mathrm{m})$, through which the tissue is inserted. The tissue is then placed on the multi-well plate, with the epidermis facing upward and the dermis submerged halfway into the culture medium. The plate is then placed in a Tedlar culture bag (Pacwill Environmental, Fredricton, Canada) to be cultured under $5 \% \mathrm{CO}_{2}$ and $95 \% \mathrm{O}_{2}{ }^{25}$ If the purpose of the culture is reepithelialization, de-epidermized dermis is attached at the center of the $10-\mathrm{mm}$ plate and then placed in each well of the 6-well plate on a cell strainer (pore size $70 \mu \mathrm{m}$, Falcon; BD Biosciences, Bedford, MAl and cultured on the air-liquid interface. ${ }^{26}$ Hence, it is possible to apply different conditions in accordance with the purpose of the culture.

In Korea, laser studies have mainly focused on the treatment efficacy, mechanism of action, and safety of lasers for pigment disorders. Currently used reconstructed skin models for the evaluation of pigment lesions include the MelanoDerm ${ }^{\mathrm{TM}}$ model, which is based on the co-culture of melanocytes and keratinocytes on a collagen membrane. The surface of the reconstructed skin is exposed to air while the bottom is maintained on the culture medium, which is mainly composed of EPI100-LLMM and EPI-100-NMM-133 that differ in terms of the culture term and the proportion of melanin-forming factors. However, no adequate model exists to evaluate the treatments response of the laser on pigment lesion. Furthermore, there are no established standard methods to induce pigment reaction by UV and other chemicals, or effective culture methods. To address the problem of short durability, human skin explants can be implanted on SCID mice, which allows months of observation. Such a model allows repetitive sampling of the tissues, and can be used to assess moisturizing barriers or other indications.

\section{ACKNOWLEDGMENTS}

This study was supported by Asan Life Science Research (2010-487).

\section{REFERENCES}

1. Lebonvallet N, Jeanmaire C, Danoux L, Sibille P, Pauly G, Misery L. The evolution and use of skin explants: potential and limitations for dermatological research. Eur J Dermatol 2010;20:671-84.

2. Kao J, Patterson FK, Hall J. Skin penetration and metabolism of topically applied chemicals in six mammalian species, including man: an in vitro study with benzo[a]pyrene and testosterone. Toxicol Appl Pharmacol 1985;81:502-16.

3. Moll I. Human skin organ culture. Methods Mol Med 2003;78: 305-10.

4. Jacobs JJ, Lehé CL, Cammans KD, Das PK, Elliott GR. Assessment of contact allergens by dissociation of irritant and sensitizing properties. Toxicol In Vitro 2004; 18:681-90.

5. Lu Z, Hasse S, Bodo E, Rose C, Funk W, Paus R. Towards the development of a simplified long-term organ culture method for human scalp skin and its appendages under serum-free conditions. Exp Dermatol 2007;16:37-44.

6. Bell E, Ehrlich HP, Buttle DJ, Nakatsuji T. Living tissue formed in vitro and accepted as skin-equivalent tissue of full thickness. Science 1981;211:1052-4.

7. Ponec M, Weerheim A, Kempenaar J, Mulder A, Gooris GS, Bouwstra J, et al. The formation of competent barrier lipids in reconstructed human epidermis requires the presence of vitamin C. J Invest Dermatol 1997;109:348-55.

8. Auxenfans C, Fradette J, Lequeux C, Germain L, Kinikoglu $B$, Bechetoille $N$, et al. Evolution of three dimensional skin equivalent models reconstructed in vitro by tissue engineering. Eur J Dermatol 2009;19:107-13.

9. Burd A, Kwok CH, Hung SC, Chan HS, Gu H, Lam WK, et al. A comparative study of the cytotoxicity of silver-based dressings in monolayer cell, tissue explant, and animal models. Wound Repair Regen 2007;15:94-104.

10. Dubertret $L$. Reconstruction of the human skin equivalent in vitro: a new tool for skin biology. Skin Pharmacol 1990;3:144-8.

11. Bernerd F. Human skin reconstructed in vitro as a model to study the keratinocyte, the fibroblast and their interactions: photodamage and repair processes. J Soc Biol 2005; 199:313-20.

12. Hantash BM, Bedi VP, Chan KF, Zachary CB. Ex vivo histological characterization of a novel ablative fractional resurfacing device. Lasers Surg Med 2007;39:87-95.

13. Thongsima S, Zurakowski D, Manstein D. Histological comparison of two different fractional photothermolysis devices operating at 1,550 nm. Lasers Surg Med 2010;42:32-7.

14. Park Gh. Histologic and molecular analysis after fractional 
laser resurfacing using skin explants [dissertation]. Ulsan: Univ. of Ulsan; 2012.

15. Bedi VP, Chan KF, Sink RK, Hantash BM, Herron GS, Rahman Z, et al. The effects of pulse energy variations on the dimensions of microscopic thermal treatment zones in nonablative fractional resurfacing. Lasers Surg Med 2007;39:145-55.

16. Hantash BM, Bedi VP, Kapadia B, Rahman Z, Jiang K, Tanner H, et al. In vivo histological evaluation of a novel ablative fractional resurfacing device. Lasers Surg Med 2007;39:96-107.

17. Manstein D, Herron GS, Sink RK, Tanner H, Anderson RR. Fractional photothermolysis: a new concept for cutaneous remodeling using microscopic patterns of thermal injury. Lasers Surg Med 2004;34:426-38.

18. Dierickx CC, Khatri KA, Tannous ZS, Childs JJ, Cohen RH, Erofeev $A$, et al. Micro-fractional ablative skin resurfacing with two novel erbium laser systems. Lasers Surg Med 2008;40:11323.

19. XuXG, Luo YJ, Wu Y, Chen JZ, Xu TH, Gao XH, et al. Immunohistological evaluation of skin responses after treatment using a fractional ultrapulse carbon dioxide laser on back skin. Dermatol Surg 2011;37:1141-9.

20. Kist DA, Elm CM, Eleftheriou LI, Studer JA, Wallander ID, Walgrave SE, et al. Histologic analysis of a 2,940 nm fractional device. Lasers Surg Med 2011;43:79-91.
21. Kim JE, Won CH, Bak H, Kositratna G, Manstein D, Dotto GP, et al. Gene profiling analysis of the early effects of ablative fractional carbon dioxide laser treatment on human skin. Dermatol Surg 2013;39:1033-43.

22. Rico PJ, Johnson TE, Mitchell MA, Saladino BH, Roach WP. Median effective dose determination and histologic characterization of porcine (Sus scrofa domestica) dermal lesions induced by 1540-nm laser radiation pulses. Comp Med 2000;50: 633-8.

23. Skovbølling Haak C, Illes M, Paasch U, Hædersdal M. Histological evaluation of vertical laser channels from ablative fractional resurfacing: an ex vivo pig skin model. Lasers Med Sci 2011;26:465-71.

24. Bonifati C, Mussi A, D'Auria L, Carducci M, Trento E, Cordiali-Fei $P$, et al. Spontaneous release of leukemia inhibitory factor and oncostatin- $M$ is increased in supernatants of short-term organ cultures from lesional psoriatic skin. Arch Dermatol Res 1998; 290:9-13.

25. Companjen AR, van der Wel LI, Wei L, Laman JD, Prens EP. A modified ex vivo skin organ culture system for functional studies. Arch Dermatol Res 2001;293:184-90.

26. Forsberg S, Rollman 0. Re-epithelialization from human skin explant cultures is promoted by ligand-activated HER3 receptor. J Dermatol Sci 2010;59:7-15. 